\title{
Surface-Sensitive, Element-Specific Magnetometry with X-Ray Linear Dichroism
}

F.O. Schumann, R.F. Willis, J.G. Tobin

This article was submitted to 1999 Symposium of the American Vacuum Society, Seattle, WA, October 18-22, 1999

\section{October 6, 1999}

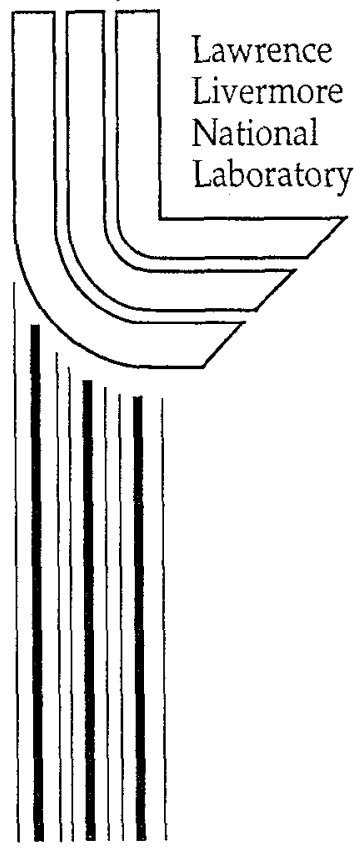




\section{DISCLAIMER}

This document was prepared as an account of work sponsored by an agency of the United States Government. Neither the United States Government nor the University of California nor any of their employees, makes any warranty, express or implied, or assumes any legal liability or responsibility for the accuracy, completeness, or usefulness of any information, apparatus, product, or process disclosed, or represents that its use would not infringe privately owned rights. Reference herein to any specific commercial product, process, or service by trade name, trademark, manufacturer, or otherwise, does not necessarily constitute or imply its endorsement, recommendation, or favoring by the United States Government or the University of California. The views and opinions of authors expressed herein do not necessarily state or reflect those of the United States Government or the University of California, and shall not be used for advertising or product endorsement purposes.

This is a preprint of a paper intended for publication in a journal or proceedings. Since changes may be made before publication, this preprint is made available with the understanding that it will not be cited or reproduced without the permission of the author.

This report has been reproduced directly from the best available copy.

Available to DOE and DOE contractors from the Office of Scientific and Technical Information

P.O. Box 62, Oak Ridge, TN 37831

Prices available from (423) 576-8401 http://apollo.osti.gov/bridge/

Available to the public from the National Technical Information Service

U.S. Department of Commerce 5285 Port Royal Rd., Springfield, VA 22161 http://www.ntis.gov/

OR

Lawrence Livermore National Laboratory Technical Information Department's Digital Library http://www.llnl.gov/tid/Library.html 


\title{
Surface-sensitive, element-specific magnetometry with $x$-ray linear dichroism
}

F.O Schumann* and R.F. Willis

The Pennsylvania State University, Department of Physics, University Park, PA 16802

\section{J.G. Tobin\#}

Department of Chemistry and Material Science, Lawrence Livermore National Laboratory, Livermore, CA 94550

\begin{abstract}
Here it is shown that the magnetic linear dichroism in x-ray photoemission (XMLD) signal can be used to measure the element specific magnetic moments in ultra thin alloy films. Comparison with recent SQUID data provides a quantitative check that demonstrates that the total magnetization derived from summing the constituent elemental moments is correct.
\end{abstract}

PACS: 75.70.Ak, 75.50.Bb, 79.60.Dp

Submitted to J.Vac.Sci. Technol. in conjunction with AVS-99.

* Present address:

Department of Chemistry and Material Science, Lawrence Livermore National Laboratory, Livermore, CA 94550

\# Correspondiing Author:

Tele 925-422-7247, Fax 925-423-7040, Email Tobin1@llnl.Gov 
1999 Symposium of the American Vacuum Society

Seattle, WA, USA, October 18-22, 1999
MI-TuA 8

Abst. \# 1358

Elementally specific magnetometry has two principal requirements: a magnetic sensitivity and an elemental specificity. By using the magnetic x-ray dichroism of core levels, one can meet these two requirements. Furthermore, by utilizing the photoemission variant of these measurements, a true surface sensitivity is obtained and many of the limitations of the absorption based techniques are avoided. It has already been shown that changes in the magnetization can be tracked elementspecifically $[1,2]$. Here, we will demonstrate using alloy systems and photoemission-based linear dichroism measurements that the dichroic differences are indeed proportional to the element specific magnetic moments.

Previously, however, a lack of consensus on what constituted the spectroscopic lineshapes induced questions concerning the applicability of this method. In this paper, we present results which show conclusively that the dichroism of the integrated sums of the spectral lineshape track the changing elemental magnetization in these alloys. Specifically, we report on the concentration dependence of the magnetic dichroism for fcc $\mathrm{Co}_{x} \mathrm{Ni}_{1-x}$ and $\mathrm{Fe}_{x} \mathrm{Ni}_{1-x}$ ultrathin alloy films of the $3 \mathrm{p}$ core levels in photoemission with linearly polarized light (XMLD). In the case of CoxNil-x alloys we have compared the dichroism obtained with circular and linearly polarized light, and observed essentially the same trends in behavior using both methods[3].

We have chosen fcc CoxNil-x and FexNil-x binary alloys because of their very different behavior in the bulk. CoxNil-x is structurally and magnetically well-behaved, in particular the magnetic moment varies linearly as a function of concentration[4]. This is in sharp contrast to fcc 
1999 Symposium of the American Vacuum Society

Seattle, WA, USA, October 18-22, 1999
MI-TuA 8

Abst. \# 1358

FexNil-x which displays a magnetic instability at about $65 \% \mathrm{\Gamma e}$ content[5]. An extended regime of fcc stabilility is possible via epitaxy on $\mathrm{Cu}(100)$ [8]. If XMLD is indeed a sensitive probe of the clement-specific magnetic moments, we should expect very different behavior for this latter alloy. This has becn obscrved and will be discussed in the following.

The growth and structural aspects of CoxNil-x and FexNil-x/Cu(100) have been thoroughly studied and we found for both systems good epitaxially pseudomorphic growth in the fcc structure [7, 8]. The sample preparation and dichroism experiments were performed at the SpectroMicroscopy Facility on Beamline 7 at the Advanced Light Source, Berkeley[9]. Ultra thin film composition was confirmed in situ using X-ray Photelectron Spectroscopy. An example of a wide scan is shown in Figure 1 , demonstrating the purity of the sample. A narrower scan, such as that shown in Figure 2, can be used to determine film concentration quantitatively. For photoemission dichroism of the $3 p$ core levels we utilized $190 \mathrm{eV}$ photons (p-polarized) and collected electrons in normal emission with an angular resolution of $2^{\circ}$. The angle of incidence of the photon beam was $60^{\circ}$ with respect to the surface normal and the magnetization was in the 'transverse' geometry[8,10]. A field pulse from a coil near the sample magnetized the sample along the 001 direction.

Next we display the concentration dependent $3 p$ magnetic dichroism asymmetry for about $6 \mathrm{ML}$ CoxNil-x/Cu(100) in fig.3 [11]. For the Co $3 p$ level we find a constant asymmetry of about $10 \%$ which agrees with previous work by Kuch et al.[12], who investigated $\mathrm{Co} / \mathrm{Cu}(100)$. For the $\mathrm{Ni}$ $3 p$ level we observe a small value of about $2 \%$. The results of the XMID measurement suggest that the magnetic states of the $\mathrm{Co}$ and $\mathrm{Ni}$ atoms are 
not changing, since their asymmetry remains constant as the stochiometry is changed. From the bulk we also know that the moments are concentration independent[4]. This might be regarded as cvidence that the XMLD asymmetry is tracking a quantity closely related to the clcmentspecific magnetic moment[2l, not merely the overall magnetization.

In an earlier publication [8], we showed the magnetic dichroism asymmetry for the $\mathrm{Fe}$ and $\mathrm{Ni} 3 \mathrm{p}$ levels. We noticed a high asymmetry for the $\mathrm{Fe} 3 \mathrm{p}$ level at low $\mathrm{Fe}$ concentrations which is strongly reduced at high Fe concentrations. As explained previously [8] we can identify three regimes (IIII) on the basis of the variation of the atomic volume and the $\mathrm{Fe}$ magnetic asymmetry[8]. Clearly the concentration dependence is now distinctively different in the FexNil-x alloy.

We want to discuss now to what extent XMLD can be used for elemental magnetometry of alloys. We were encouraged to pursue this aspect of our work by the results of Sirotti et al. on Fe bulk samples[2]. They compared the low-temperature dependence of the $\mathrm{Fe} 3 p$ linear dichroism and asymmetry in the the spin-polarization of secondary electrons. The latter is now very well established to be proportional to the magnetization[13]. Sirotti et al. observed that both techniques gave identical results and they concluded that the XMLD asymmetry is indeed proportional to the overall magnetization in their one element system.

As shown in fig. 3 , the element-specific magnetic properties of Co and $\mathrm{Ni}$ in CoxNil-x remain constant. From these data, we find that the average dichroism asymmetry for $\mathrm{Co}$ and $\mathrm{Ni}$ is $9.8 \%$ and $2 \%$, respectively. We can replot the data of fig.l by 'calibrating' the average asymmetries in the following way: (i) $9.8 \%$ asymmetry equals 1.7 Bohr Magnetons for Co 
and (ii) 2\% asymmetry equals 0.6 Bohr Magnetons for $\mathrm{Ni}$. These are the known magnetic moments for bulk $\mathrm{Co}$ and $\mathrm{Ni}[4,14]$. Now we are able to calculate the average moment as a function of concentration for each data point. First we convert the dichroism asymmetry for $\mathrm{Co}$ and $\mathrm{Ni}$ into an element-specific moment. Sccondly we calculate the average moment using the following stochiometric equation:

$$
\mu=x \cdot \mu_{C o l F e}(x)+(1-x) \cdot \mu_{N i}(x)
$$

If we replace the actual data points with the averaged dichroism asymmetry for $\mathrm{Co}$ and $\mathrm{Ni}$, the solid line results, plotted in fig. 4. The error bars reflect the uncertainties of the original data in fig.3.

We can repeat this procedure for FexNil-x alloys, for which we use the following " calibration": (i) $8.5 \%$ asymmetry equals 2.5 Bohr Magnetons for $\mathrm{Fe}$ and (ii) $2 \%$ asymmetry equals 0.6 Bohr Magnetons for Ni. In Ref 8., we have shown that the phase diagram of FexNil-x alloys can be divided into 3 regimes. We concluded that $\mathrm{Fe}$ is in a high-spin (HS) state for concentrations up to about $65 \%$. Following the work of Abrikosov et al. we associate this state with an atomic magnetic moment of 2.5 Bohr Magnetons; for $\mathrm{Ni}$ we have used again the bulk value[15]. Again we can calculate the average moment using cquation (1). The solid line in fig.5 is the result if we replace the actual data points by the fitted curve, analogous to fig.4.

Clearly in order to endorse XMLD as an element-specific magnetometer we need a comparison to results using absolute 
magnetometer. Recently Freeland et al. provided results to this effect on ultrathin FexNil-x alloys[16]. They investigated 4 ML thick FexNil-x multilayers grown mainly on a $\mathrm{Cu}(111)$ substrate. Using SQUID magnetometry they have determined absolute values of the average moment. We show their results together with our reploted data from Ref 8 . in Fig. 5. We have also included also a data point for $6 \mathrm{ML} \mathrm{Fe} / \mathrm{Cu}(100)$ from a previous publication of the same group[17]. This clearly shows an almost linear increase for $\mathrm{Fe}$ concentrations up to about $65 \%$. The slope of the curve extrapolates to a value of about 2.9 Bohr Magnetons representing fcc $\mathrm{Fe}$ in its HS state. Further evidence that the Fe moment stays constant in a HS state up to $65 \%$. Fe has been provided by Mossbauer spectroscopy [16]. This work confirms our conclusions based on the high value of the $\mathrm{Fe}$ asymmetry up to about $65 \% \mathrm{Fe}$ content as discussed previously[8].

Going beyond $65 \%$ Fe content Freeland et al. observe a strong reduction of the magnetic moment towards smaller values. For pure $\mathrm{Fe}$ they find a magnetic moment of about 1 Bohr Magnetons. In this Fe-rich regime, their data show a considerable scatter of values, presumably due to different amounts of strain.This is particularly true for the (100) oriented thin films. These show a consistently smaller value of the moment than those in the (111) orientation.

The apparent discrepancy between the experimental data points and our work (full line) in fig.5 can be explained as follows. The difference between the 2 data points from samples with the (100) orientation is due to the thickness. Increasing the thickness obviously reduces the average moment due to the relief of volumetric strain, which is consistent with the Mossbauer experiments on $\mathrm{Fe} / \mathrm{Cu}(100)$ [18]. Thickness dependent magnetic 
1999 Symposium of the Amcrican Vacuum Socicty

Seattle, WA, USA, October 18-22, 1999
MI-TuA 8

Abst. \# 1358

and structural properties are known to exist in the thickness regime 4-7 Ml. [18-21]. This is also true for FexNil-x $/ \mathrm{Cu}(100)$ alloy films as previously reported [8]. We suspect that the FexNil-x(111) films investigated by Freeland et al. have not the same atomic volume as our films for $\mathrm{Fe}$ concentrations larger than $65^{\prime} \%$. Either the films in the (111) direction have not fully relaxed or there is real difference in the volume instability. As it turns out there is evidence for the latter. The structure of $\mathrm{Fe} / \mathrm{Cu}(111)$ has been carefully determined by means of LEED I-V [22]. In accord with earlier reports [23] it is found that for thicknesses up to $5 \mathrm{ML}$ a fcc phase exists and we derive an atomic volume of 11.68 Angstroms-cubed for 5 ML. This value is almost exactly in between 11.4 and 12.1 Angstroms cubed which are representative for 2 different magnetic phases in the case of $\mathrm{Fe} / \mathrm{Cu}(100)$ [211. We have essentially derived the same values via an extrapolation [8]. Following the work of Keavney et al. we associate an atomic volume of $11.4 \mathrm{~A} 3$ with an average $\mathrm{Fe}$ moment of $0.3 \mathrm{Bohr}$ Magnetons. On the other hand an average moment of about 2 Bohr Magnetons has been observed for the HS state[17, 18]. Making the reasonable assumption that the moment is a linear function of the atomic volume[17] we determine an average moment of about 1 Bohr Magnetons for fcc $\mathrm{Fe} / \mathrm{Cu}(\mathrm{lll})$. This is in good agreement with the results of Freeland et al.[16].

It is also important to note that Tian et al. do not observe extra spots in the fcc phase contrary to the observation for fcc $\mathrm{Fe} / \mathrm{Cu}(100)[19,21,24]$. Obviously the structural instabilities and therefore magnetic states do not manifest themselves as clearly for pure $\mathrm{Fe} / \mathrm{Cu}(111)$ as compared to $\mathrm{Fe} / \mathrm{Cu}(100)$. Our work and the results of Freeland et al. agree that for up to 
$65 \% \mathrm{Fe}$ content, $\mathrm{Fe}$ is in a HS state. Beyond this concentration the behavior of the atomic volume for FexNil-x in the (100) and (111) orientation i.s different.

In summary, we have systematically investigated element-specific magnetic properties with XMLD for two different binary alloys. These results endorse the use of XMLD in photoemission as an element-specific technique for studying ferromagnetism in metastable, binary-alloy, epitaxial films. The difference in the integrated spectral lineshapes when the direction of the magnetization is switched reflects the magnitude of the magnetization. This is clearly reflected in the contrasting behavior of the CoxNil-x and FexNil-x alloys with changing stochiometry. The observed behavior of the ferromagnetic response suggests a simple summing of the magnetic moments, depending on the alloy composition. This in turn, further underlines the elementally specific magnetometry deduced from the XMLD signals.

\section{Acknowledgments}

Funding was provided via NSF grant DMR-95-21126. This work was also performed under the auspices of the U.S. Department of Energy by LLNL under contract No. W-7405-ENG-48. We would like to acknowledge support with the ALS measurements from Drs. K. Goodman, J. Denlinger, E. Rotenberg and A. Warwick. 
1999 Symposium of the American Vacuum Society

Seattle, WA, USA, October 18-22, 1999
MI-TuA 8

Abst. \# 1358

\section{References}

[1.] D. Schmitz, O. Rader, C. Carbone, and W. Eberhardt, Phys. Rev B, 54, 15352 (1996).

[2.]F. Sirotti, G. Panaccione, and G. Rossi, Phys. Rev. B, 52, R17063 (1995).

[3.] S.Z. Wu, et al., J. Vac. Sci. Technol., A 15, 2287 (1997).

[4.] M.F. Collins and D.A. Wheeler, Proc. Phys. Soc., 82, 633 (1963)

[5.] E.F. Wassermann, J. Mag. Magn. Mat., 100, 346 (1991).

[6.] J. Dresselhaus, M. Moller, T. Kleemann, and E. Kisker, J. Mag. Magn.

Mat., 148,172 (1995).

[7] F.O. Schumann, S.Z. Wu, G.J. Mankey, and R.F. Willis, Phys. Rev. B, 56, 2668 (1997).

[8.] F.O. Schumann, R.F. Willis, K.G. Goodman, and J.G. Tobin, Phys. Rev. Lett., 79, 5166 (1997).

[9. J.G. Tobin, et al., J. Appl. Phys., 79, 5626 (1996).

[10.] C. Roth, F.U. Hillebrecht, H.B. Rose, and E. Kisker, Phys. Rev. Lett., 70,3479 (1993)

[11.] The element-specific asymmetry is calculated as the ratio of the difference spectrum over twice the mean peak height. We can not use the expression $\mathrm{I}($ up)-I(down)/I(up) $+\mathrm{I}($ down $)$ due to the concentration dependence of the $3 \mathrm{p}$ peak heights.

[12.] W. Kuch, et al., Phys. Rev. B, 51, 609 (1995).

[13.] H.C. Siegmann, J. Phys.: Condens. Matter, 4, 8395 (1992).

[14.] C. Kittel, Introduction to Solid Stnte Physics 1991, New York, Chichester, Brisbane, Toronto, Singapore: John Wiley \& Sons, Inc. 
1999 Symposium of the American Vacuum Society Seattle, WA, USA, October 18-22, 1999
MI-TuA8

Abst. \# 1358

[15.] I.A. Abrikosov, et al., Phys. Rev. B, 51,1058 (1995).

[16.]J.W. Frecland, I.L. Grigorov, and J.C. Walker, Phys. Rev. B, 57, 8() (1998).

[17.]D.J. Keavney, et al., Phys. Rev. Lett., 74, 4531(1995).

[18.]R.D. Ellerbrock, et al., Phys. Rev. Lett., 74, 3053 (1995).

[19.]J. Thomassen, et al, Phys. Rev. Lett., 69(26), 3831 (1992).

[20.]M. Zharnikov, et al, Phys. Rev. Lett., 76, 4620 (1996).

[21.]S. Muller, et al., Phys. Rev. Lett., 74, 765 (1995).

[22.]D. Tian, F. Jona, and P.M. Marcus, Phys. Rev. B, 45,11216 (1992).

[23.]W. Kummerle and U. Gradmann, Solid State Comms., 24, 33 (1977).

[24.]J. Thomassen, B. Feldmann, and M. Wuttig, Surf.Sci., 264, 406 (1992). 
1999 Symposium of the American Vacuum Society

Seattle, WA, USA, October 18-22, 1999
MI-TuA 8

Abst. \# 1358

\section{Figure captions}

Fig.1: Wide scan of the $\mathrm{Co39Ni61/Cu(001)}$ sample, using $\mathrm{Mg} \mathrm{K}$-alpha radiation for excitation.

Fig.2 A narrower XPS scan looking at the $2 \mathrm{p}$ peaks of $\mathrm{Cu}(952,933 \mathrm{eV})$, $\mathrm{Ni}(870,852 \mathrm{eV})$ and $\mathrm{Co}(793,778 \mathrm{eV})$. Elemental concentrations are determined from scans such as this.

Fig.3: Concentration dependence of the Co (points) and $\mathrm{Ni}$ (squares) 3p asymmetry for $6 \mathrm{ML}$ thick CoxNil-x films at $300 \mathrm{~K}$.

Fig.4: Average moment of CoxNil-x $/ \mathrm{Cu}(100)$ alloy films using the 'calibration' as explained in the text.

Fig.5: Avcrage moment of FexNil-x/Cu(100) alloy films using the 'calibration' as explained in the text. Nlso shown is a comparison of the calibrated curve and the results of Freeland et al.( points/open squares are for $(111) /(100)$ orientation) [16]. Included is also data point from Keavney et al. (square)[17]. Triangle follows from Kummerle et al. on $\mathrm{Fe} / \mathrm{Cu}(111)$ [23]. 


\section{Co39Ni61}

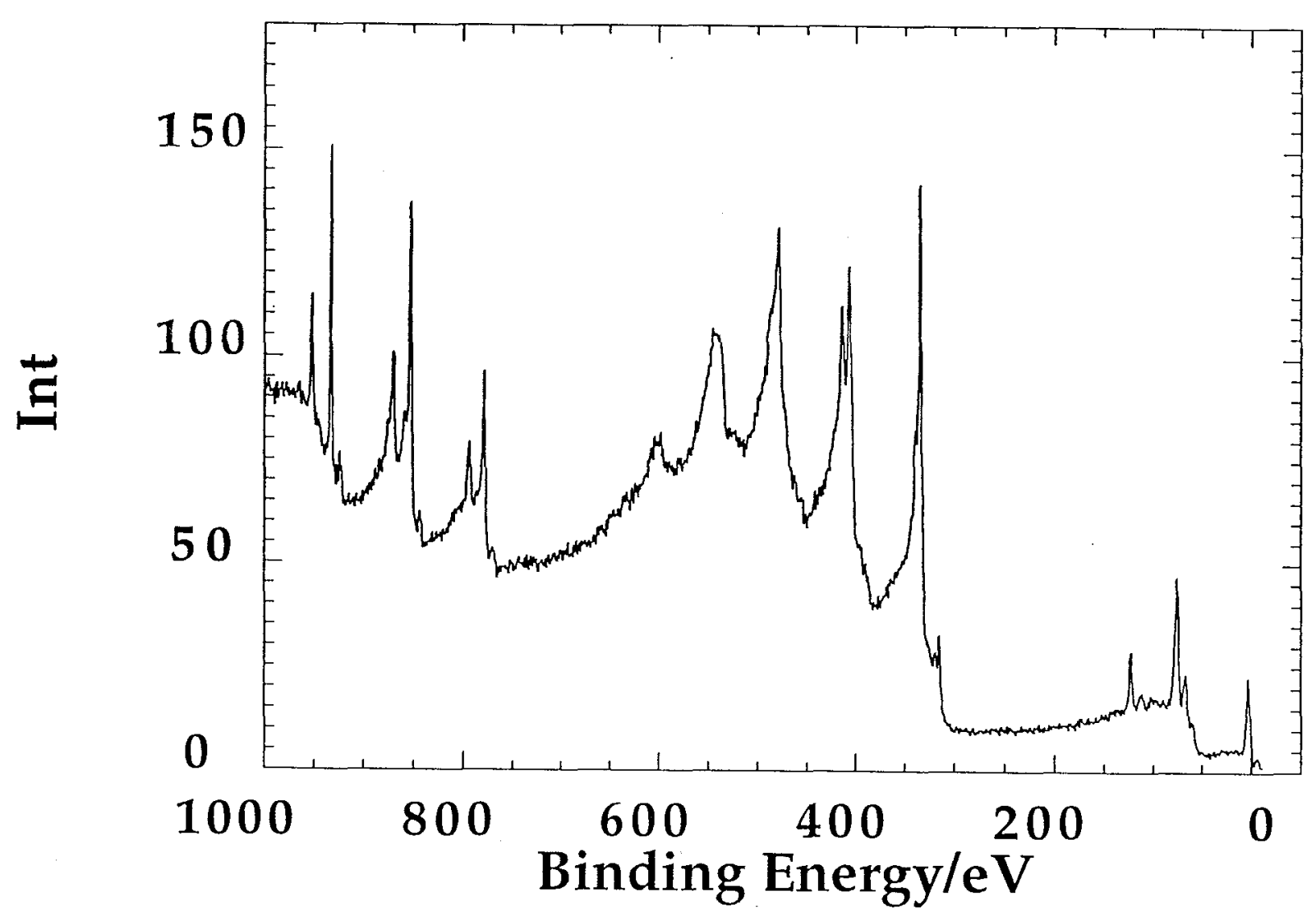

Fig.1

F.O. Schumann et al. 
1999 Symposium of the American Vacuum Society Seattle, WA, USA, October 18-22, 1999
MI-TuA 8

Abst. \# 1358

normalized peak ratio $\mathrm{Co} / \mathrm{Co}+\mathrm{Ni}: \quad 04 / 10.635$

0.34 Co $0.66 \mathrm{Ni}$

corrected with respect to cross section and mean free path (factor 1.07-1.03) result: $39 \% \mathrm{Co}$

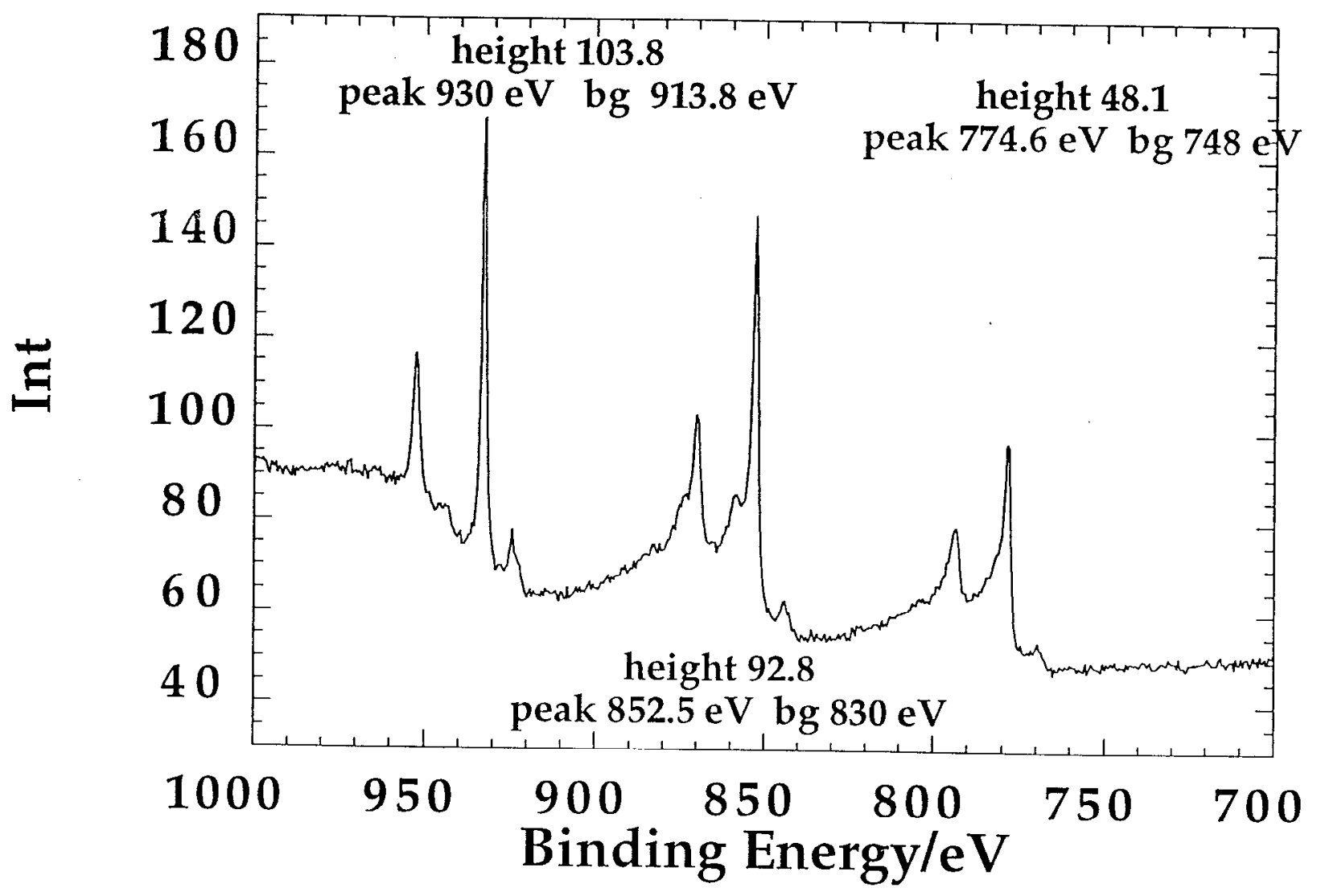

Fig.2

F.O. Schumann et al. 
1999 Symposium of the American Vacuum Society

Seattle, WA, USA, October 18-22, 1999
MI-TuA 8

Abst. \# 1358

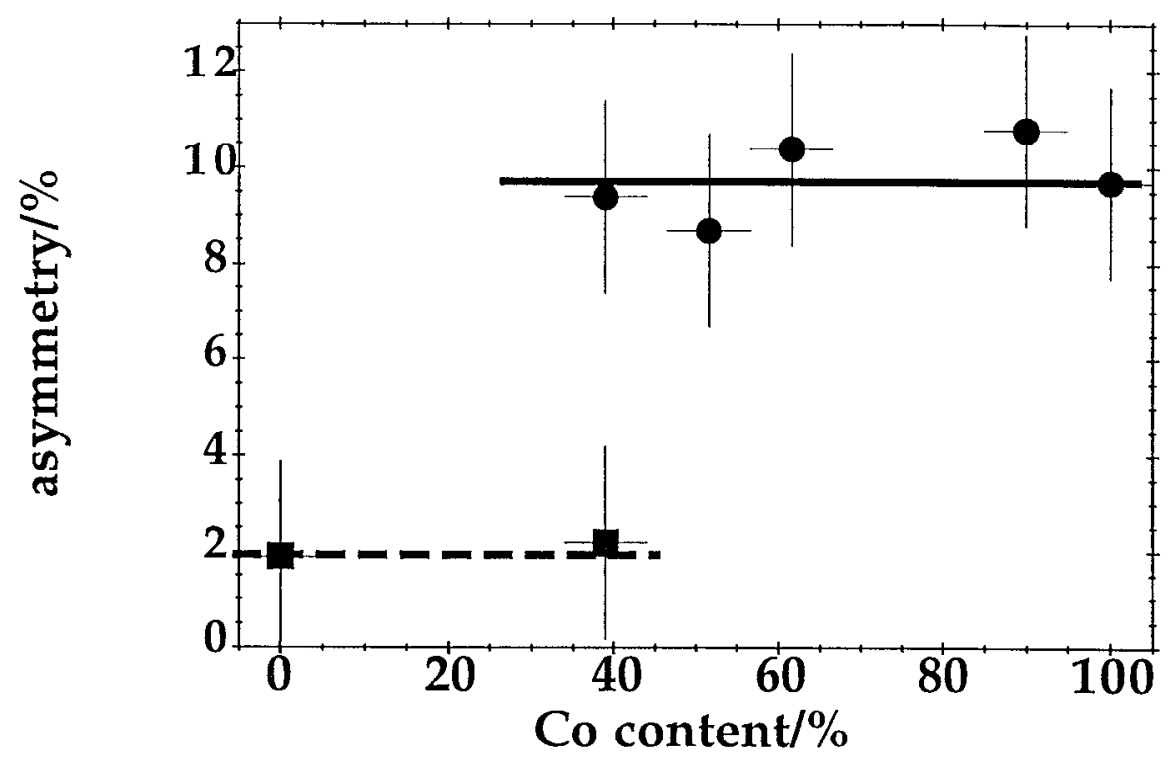

Fig.3

F.O. Schumann et al. 
1999 Symposium of the American Vacuum Society

Seattle, WA, USA, October 18-22, 1999

MI-TuA 8

Abst. \# 1358

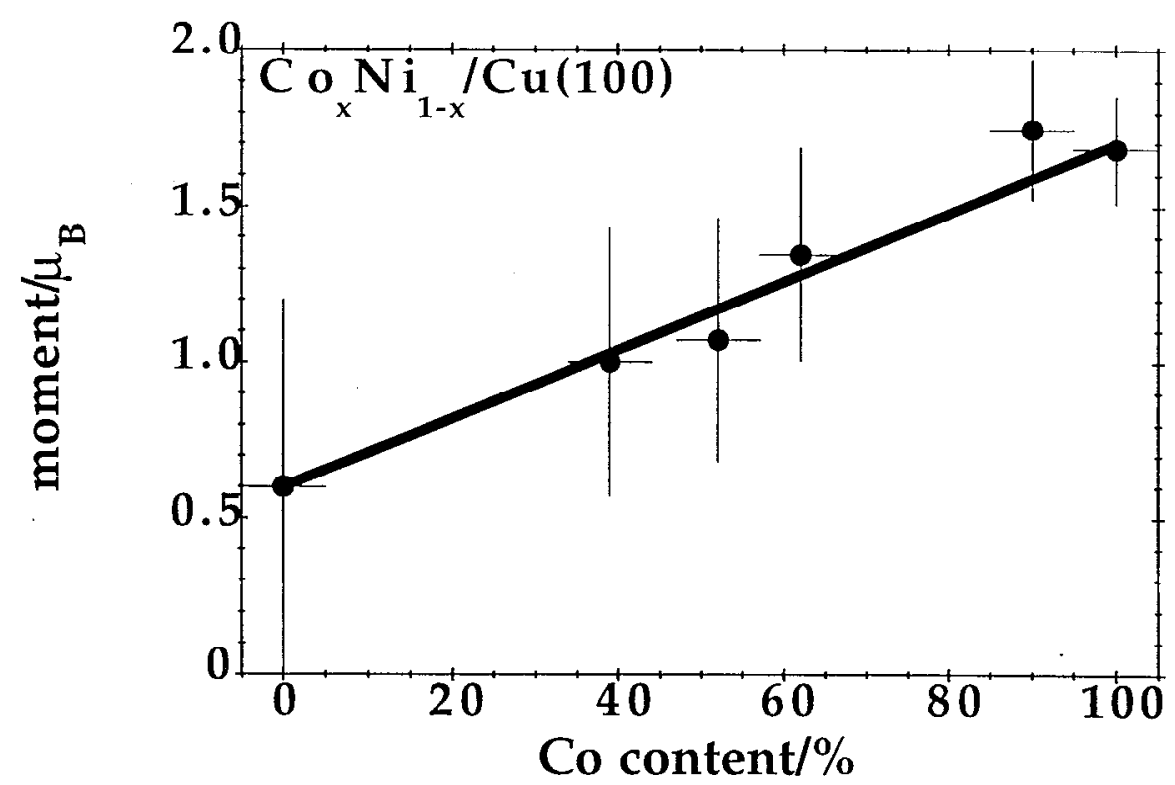

Fig.4

F.O. Schumann et al. 
1999 Symposium of the American Vacuum Society

Seattle, WA, USA, October 18-22, 1999
MI-TuA 8

Abst. \# 1358

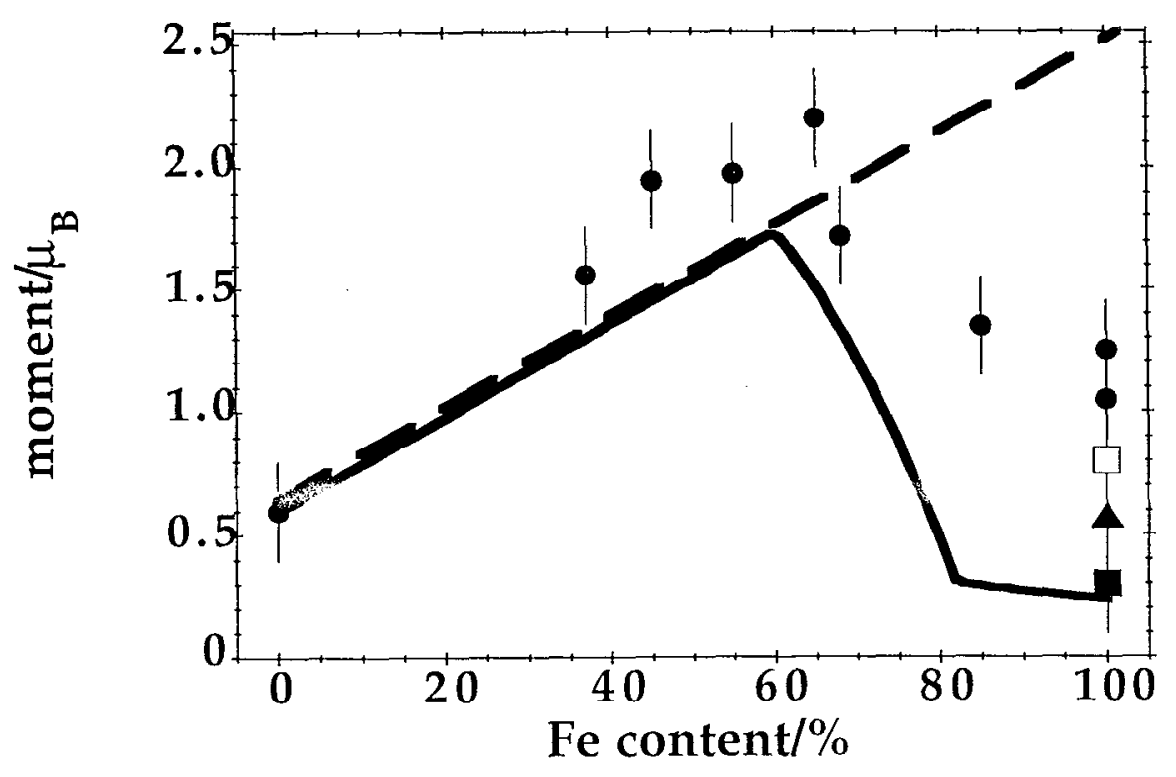

Fig.5

F.O. Schumann et al. 